\title{
Overview of Interferometer-Type Gravitational Wave Detectors
}

\author{
David E McClelland \\ Department of Physics, Faculty of Science, The Australian Naiotnal \\ University, Canberra, 0200, Australia
}

\begin{abstract}
Within the next decade giant laser interferometers should detect gravitational waves. Here we present an overview of these instruments including both ground and spaced based antennae.
\end{abstract}

\section{Introduction to Gravitational Waves}

Gravitational waves, or ripples in the curvature of space-time, are predicted by Einstein's General Theory of Relativity. They are quadrupolar in nature and propagate at the speed of light. Their existence has been confirmed by observing the energy loss of the binary pulsar PSR1913+16 due to gravitational wave emission. Figure la shows measurements of the shift of periastron time as a function of time (Taylor \& Weinberg 2000). The points are the measurements with error bars too small to show up on this plot. The curve is the prediction based on gravitational wave emission. Hulse \& Taylor won a Nobel Prize for this work in 1993. Today, the direct detection of gravitational radiation remains a major goal of fundamental physics.

Gravitational waves (GW) are emitted by the most violent events in the universe, provided they exhibit some non spherical geometry. As summarized by Schutz in this volume, these include compact binary in spirals and mergers, supernovae, pulsars, stochastic sources such as the relic spectrum left over from the Big Bang and undiscovered events which have no electromagnetic signature. As depicted in Figure 1b, the GW spectrum covers many orders of magnitude. Ground-based detectors such as LIGO (LIGO 2003) and VIRGO (VIRGO 2003) explore the audio spectrum from $10 \mathrm{~Hz}$, whilst antennae in space such as LISA (LISA 2003), will be sensitive in the frequency range from $0.1 \mathrm{mHz}$ to $1 \mathrm{~Hz}$.

\section{Detection using Laser Interferometry}

The most promising technology for gravitational wave detection is long baseline laser interferometry. A passing gravitational wave will alternately stretch then contract one arm of a Michelson interferometer whilst contracting then stretching the other arm. The problem is that the effect is extremely small: expressed as a relative length change, $\delta L / L$, it is of the order of $10^{-22}$. Whilst for the groundbased detectors, the interferometer arms contain mirrors to reflect light, LISA uses a transponder based system in which the incoming beam from a distant spacecraft is detected, the signal then used to phase lock a second laser, which sends light back to the first spacecraft. 

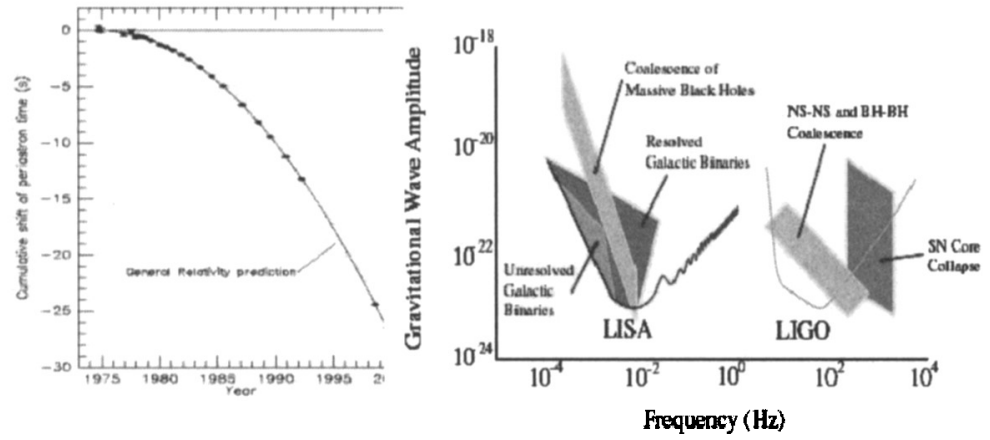

Figure 1. (a) Comparison between observations of the binary pulsar PSR1913+16 and the predictions of general relativity (b) Gravity wave frequency band.
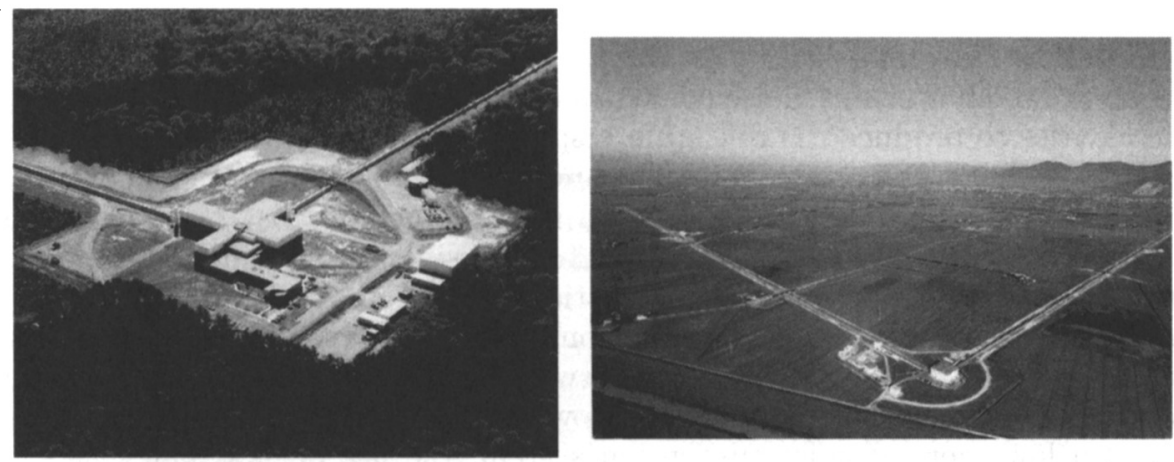

Figure 2. (a) LIGO site Hanford Washington (b) VIRGO site Cascina.

\subsection{Ground-Based Detectors}

Current Currently there are 4 funded mid to long baseline interferometer projects: the US LIGO Project (Figure 2a); the French/Italian VIRGO Project (Figure 2b); the German/British collaboration GEO600 (GEO 2003), and the Japanese Project TAMA300 (TAMA 2003). Based in the main part on technology developed early last decade, the first generation of large instruments (LIGO/VIRGO) are predicted to reach a spectral sensitivity of $10^{-22} / \sqrt{ } \mathrm{Hz}$ between $50 \mathrm{~Hz}$ and $1000 \mathrm{~Hz}$ by the year 2004. The optical layout for the long baseline first generation detectors is shown in Figure 3a. It consists of a standard Michelson interferometer with arm cavities to increase the sensitivity to phase change, and a power recycling mirror to build up the laser power thereby decreasing shot noise. As shown in Figure 3b, LIGO has already achieved a sensitivity on the order of $2.5 \times 10^{-22} / \sqrt{ } \mathrm{Hz}$ at $200 \mathrm{~Hz}$ (LIGO 2003).

Typically such interferometers are limited at low frequency (below $40 \mathrm{~Hz}$ ) by seismic noise (ultimately, below $4 \mathrm{~Hz}$, noise from direct Newtonian coupling of the interferometers mirrors to the environment). Midband noise is thermal 

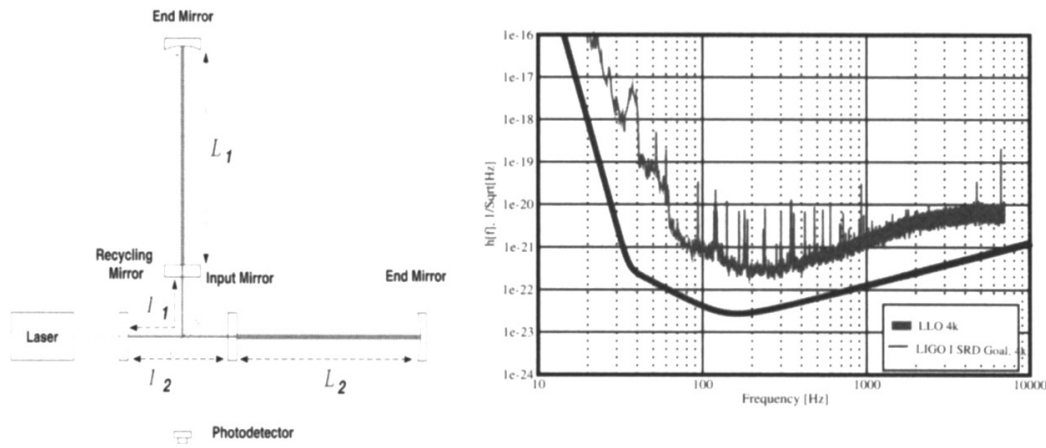

Figure 3. (a) Optical layout of first generation interferometers (b) Measured LIGO sensitivity curve 2003.

noise due to Brownian motion in the test masses and their suspension; above $200 \mathrm{~Hz}$ or so, photon shot noise combines with the averaging of the signal in the arm cavities to produce the roll up in response shown in Figure 1b.

The Future To ensure detection and the birth of gravitational wave astronomy the sensitivity of ground-based interferometers must be improved by at least a factor of order 10 , increasing the accessible volume of the universe by 1000 times. Toward this end, an international R\&D effort on the four main detector subsystems (Laser and Optics; Configurations; Isolation, Suspension and Thermal Noise; Data Analysis) is underway. The key elements of an upgrade are (LSC 1999): an increase in laser power to greater than 100W; the adoption of a low thermal noise suspension system; the use of new materials such as sapphire for the test masses; the implementation of a signal recycling optical configuration; and possibly, cooling of the test masses (LCGT 2003).

\section{Space based Detectors}

The Laser interferometer Space Antenna (LISA) consists of 3 spacecraft (see Figure 4a) separated by 5 million $\mathrm{km}$, in solar orbit trailing the earth by 20 degrees. The spacecraft roll on a cone of half angle 60 degrees with each moving in a slightly elliptic and inclined orbit around the sun. This configuration ensures that: the spacecraft face the sun at a constant angle giving a stable thermal environment; the orbits of the 3 spacecraft form a stable configuration; the center of the spacecraft triangle trails the earth by 20 degrees (or 50 million $\mathrm{km}$ ) ensuring that the distance to the earth is quite stable for radio communication.

The LISA sensitivity curve (see Figure 1b) is low frequency limited by acceleration noise such as noise, not shielded by the drag free system, from gravitating masses on the craft when temperature changes their distances; charging of the test masses due to cosmic radiation; and residual gas in test mass housing. Shot noise limits the sensitivity between $1 \mathrm{mHz}$ and $10 \mathrm{mHz}$ with only a few nanowatts of light collected by the telescope on the distant space craft. The sensitivity rolls off at high higher frequencies (above $10 \mathrm{mHz}$ ) due to averaging out of the signal. 

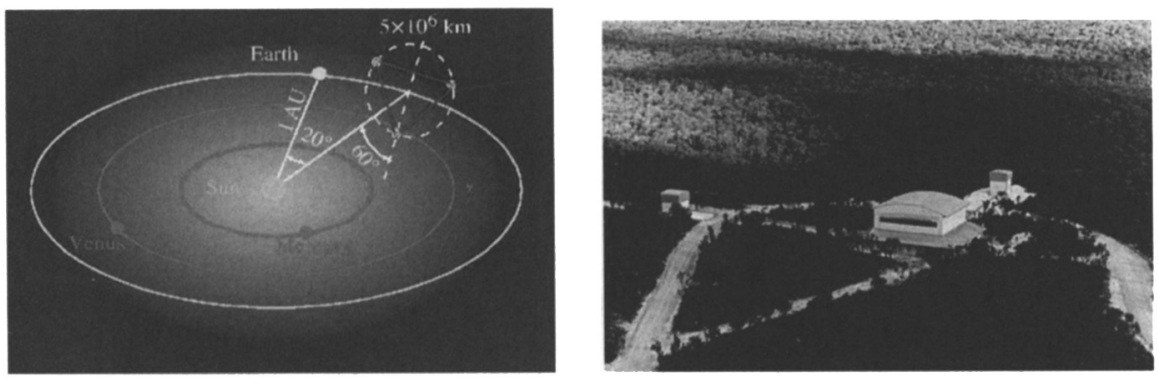

Figure 4. (a) orbit of the LISA spacecraft (b) ACIGA Facility, Gingin

LISA is planned for launch in 2011. Due to the enormous cost of the LISA mission (estimated at greater than $\mathrm{A} \$ 2$ billion in total), it is a combined ESANASA project. The development of space based trials of key LISA hardware is well advanced with the SMART-2 mission planned for launch in 2006.

\section{Australia's role to date}

The Australian Consortium for Interferometric Gravitational Astronomy (ACIGA; ACIGA 2003) carries out research on all detectors subsystems. Its facility in Gingin in Western Australia (Figure $4 \mathrm{~b}$ ), at $80 \mathrm{~m}$ in length, is an ideal instrument for integrating next generation technologies associated with high power interferometry.

Acknowledgments. I would like to thank LIGO, VIRGO, GEO600, LISA, and TAMA300 for making pictures and other information available for this overview.

\section{References}

Taylor, J.H. and Weinberg, J.M. 2000, unpublished LIGO 2003, http://www.ligo.caltech.edu VIRGO 2003, http://www.virgo.infn.it LISA 2003, http://sci.esa.int/science-e/www/area/index.cfm? fareaid $=27$, LISA 2003, http://lisa.jpl.nasa.gov GEO 2003, http://www.geo600.uni-hannover.de TAMA 2003, http://tamago.mtk.nao.ac.jp LSC 1999, LSC White Paper on Detector R \& D, http://www.ligo.org LCGT 2003, http://www.icrr.u-tokyo.ac.jp/gr/gre.html ACIGA 2003, http://www.anu.edu.au/Physics/ACIGA 